\title{
Variable oxygen environments and DNMT2 determine the DNA cytosine epigenetic landscape of Plasmodium falciparum
}

Elie Hammam ${ }^{1^{*}}$, Samia Miled ${ }^{2 *}$, Frédéric Bonhomme ${ }^{3}$, Benoit Arcangioli ${ }^{4}$, Paola B.

$$
\text { Arimondo }^{3}, \text { Artur Scherf }^{1, \#}
$$

${ }^{1}$ Institut Pasteur, Université de Paris, Biology of Host Parasite Interaction Unit, INSERM 1201, CNRS ERL9195, 75724 Paris, France

${ }^{2}$ Université de Paris, CNRS, Institut Jacques Monod, 75013 Paris, France, École Technique Supérieure du Laboratoire, 75013 Paris, France

${ }^{3}$ Institut Pasteur, Université de Paris, Epigenetic Chemical Biology, UMR 3523, CNRS, 75724 Paris, France

${ }^{4}$ Institut Pasteur, Université de Paris, Genome Dynamics Unit, UMR 3525 CNRS, 75724

Paris, France

*co-first authors

\#Correspondance to: artur.scherf@pasteur.fr 


\section{Abstract}

DNA cytosine methylation and its oxidized products are important epigenetic modifications in mammalian cells. Although 5-methylcytosine $(5 \mathrm{mC})$ was detected in the human malaria parasite Plasmodium falciparum, the presence of oxidized $5 \mathrm{mC}$ forms remain to be characterized. Here we establish a protocol to optimize nuclease-based DNA digestion for the extremely AT-rich genome of $P$. falciparum $(>80 \% A+T)$ for quantitative LC-MS/MS analysis of 5-hydroxymethylcytosine (5hmC), 5-formylcytosine $(5 \mathrm{fC})$ and 5-carboxylcytosine $(5 \mathrm{caC})$. We demonstrate the presence of $5 \mathrm{hmC}, 5 \mathrm{fC}$ and $5 \mathrm{caC}$ cytosine modifications in a DNMT2-only organism and observe striking ratio changes between $5 \mathrm{mC}$ and $5 \mathrm{hmC}$ during the 48-hour blood stage parasite development. Parasite-infected red blood cells cultured in different physiological oxygen concentrations revealed a shift in the cytosine modifications distribution towards the oxidized $5 \mathrm{hmC}$ and $5 \mathrm{caC}$ forms. In the absence of the canonical C5-DNA methyltransferase (DNMT1 and DNMT3A/B) in P. falciparum, we show that all cytosine modifications depend on the presence of DNMT2. We conclude that DNMT2 and oxygen levels are critical determinants that shape the dynamic cytosine epigenetic landscape in this human pathogen. 


\section{Introduction}

Since its discovery in $1948^{1}, 5$-methylcytosine $(5 \mathrm{mC})$ in DNA, also known as the 5 th base of the genome, is now the best characterized epigenetic mark in higher eukaryotes, playing very important functions in different cellular processes such as cell differentiation and $\mathrm{X}$ chromosome inactivation ${ }^{2,3}$. DNA cytosine methylation is catalyzed by members of the DNA methyltransferases family (DNMT1 and DNMT3A/B) $)^{4-6}$ and $5 \mathrm{mC}$ is mostly found in $\mathrm{CpG}$ rich regions of gene promoters (CpG islands or CGls) ${ }^{7-9}$. The presence of $5 \mathrm{mC}$ within CGls of promoter regions is often linked with the inactivation of the corresponding gene $e^{10,11}$. This occurs through the impairment of the binding of transcription factors and the recruitment of methyl $\mathrm{CpG}$ binding proteins (MBDs) creating a transcriptionally repressive environment ${ }^{12}$.

Besides $5 \mathrm{mC}$, another major DNA cytosine modification in eukaryotes is 5hydroxymethylctosine $(5 \mathrm{hmC})^{13}$. $5 \mathrm{hmC}$ is generated as the first oxidative product of the TET (Ten eleven translocation)-dependent DNA cytosine demethylation pathway ${ }^{14}$. TET enzymes (namely TET1, TET2 and TET3 in mammalian cells ${ }^{15}$ ) progressively oxidize $5 \mathrm{mC}$ into $5 \mathrm{hmC}$ and further into $5 \mathrm{fC}$ (5-formylcyotsine) and 5caC (5-carboxylcytosine) ${ }^{15}$. These latter can be actively removed by different mechanisms including the activity of a thymine DNA glycosylase (TDG) coupled with the base excision repair machinery (BER) or passively lost upon DNA replication ${ }^{14}$. It is now well established that $5 \mathrm{hmC}$ is on its own an independent epigenetic mark in particular in the $\operatorname{brain}^{16,17}$. In addition, it has been suggested that $5 \mathrm{fC}$ and $5 \mathrm{caC}$ may also have regulatory functions especially when present at the promoters of highly expressed genes ${ }^{18,19}$. Importantly, TET proteins require cofactors for their activity such as alpha-ketoglutarate, iron and oxygen $\left(\mathrm{O}_{2}\right)^{20}$. The latter is a very important factor that modulates the activity of TET enzymes such as in tumours ${ }^{21,22}$. 
DNA cytosine modifications have also been reported for the extremely AT-rich genome $(>80 \%)$ of the protozoan malaria parasite $P$. falciparum ${ }^{23,24}$. This important human pathogen is exposed to fluctuating oxygen levels during its complex life cycle in the human host (liver and blood) and Anopheles mosquito vector (midgut and salivary glands). While circulating parasite infected erythrocytes (IE) are exposed to different $\mathrm{O}_{2}$ levels in patients that vary between $5 \%$ in venous blood and $13 \%$ in arterial blood ${ }^{25}$, these levels can reach up to $20-21 \%$ in the mosquito vector, where the tracheal system enables transport of gaseous oxygen from the atmosphere directly to the inner organs ${ }^{26}$. Fluctuating $\mathrm{O}_{2}$ levels could constitute a sensor system which participates in a rapid adaptation of $P$. falciparum to its immediate cellular environment.

In this study we analyzed if different oxygen level environments encountered during the life cycle of $P$. falciparum can impact DNA cytosine methylation, $5 \mathrm{mC}$, and its oxidative derivatives, $5 \mathrm{hmC}, 5 \mathrm{fC}$ and $5 \mathrm{caC}$. To this end, we used ultra-performance liquid chromatography-tandem mass spectrometry (LC/MS-MS) on digested genomic DNA for the identification and quantification of $5 \mathrm{mC}$ and its oxidative derivatives for an extremely AT-rich genome. We showed that DNA cytosine $5 \mathrm{mC} / 5 \mathrm{hmC}$ ratio is dynamically regulated throughout the 48-hour asexual blood stage parasite progression with a sharp increase of $5 \mathrm{mC}$ at the trophozoite stage. Importantly, hyperoxic culture conditions led to a dramatic shift of DNA cytosine modifications towards the oxidative derivatives $5 \mathrm{hmC}$ and 5caC. Given the absence of the DNA methyltransferase family genes DNMT1 and DNMT3A/B, malaria parasites belong to a small group of DNMT2-only organisms ${ }^{27} . P$. falciparum DNMT2-mediated tRNA methylation is not essential during standard in vitro culture conditions but was shown to maintain cellular homeostasis in the presence of different stressors ${ }^{28}$. In this work we establish that plasmodial DNA cytosine modifications depend on the presence of DNMT2. The observed dual specificities of this enzyme as 
methyltransferase for DNA (this work) and tRNA ${ }^{28}$ points to an adaptative evolutionary process in malaria parasites. Our findings expand the plasmodial epigenetic marks to three cytosine $5 \mathrm{mC}$ oxidative modifications and establish a direct link between variable oxygen environments encountered during the life cycle and the DNA cytosine modification pathway in malaria parasites.

\section{Materials and methods}

\section{P. falciparum and mESC in vitro culture}

P. falciparum 3D7-wildtype and Pf-DNMT2KO clones 1 and 2 parasites were maintained in culture and synchronized as previously described ${ }^{29}$. Parasites were routinely cultured at $5 \%$ normoxic $\mathrm{O}_{2}$ levels and for specific experiments at $20 \%$ hyperoxic $\mathrm{O}_{2}$ levels keeping $\mathrm{CO}_{2}$ levels well controlled at $5 \%$. For genomic DNA preparation, parasites were first lysed using $0.15 \%$ saponin and parasite pellets were used to extract gDNA for digestion and hydrolysis.

mESC cells were cultured in standard mouse ES medium supplemented with LIF (1000 $\mathrm{U} / \mathrm{mL}$, Life Technologies) to prevent differentiation, and incubated at $37^{\circ} \mathrm{C}, 5 \% \mathrm{CO} 2$ as describe earlier ${ }^{30}$.

\section{Genomic DNA extraction}

gDNA from parasites and mESC pellets was prepared using phenol-chloroform extraction. Briefly, $100 \mu \mathrm{L}$ of DNA Extraction Buffer and $15 \mu \mathrm{L}$ of Proteinase $\mathrm{K}$ from Thermo Fisher (\# EO0491) were added to 100ng of gDNA. The tubes were mixed and incubated at $55^{\circ} \mathrm{C}$ overnight. Equal volume of Phenol/Chloroform ( $\mathrm{pH} \mathrm{8.0)}$ was added to the digested sample. The tubes were then spun for $5 \mathrm{~min}$ at $15.000 \mathrm{rpm}$ separating the phases into the aqueous and the organic. For each sample, $650 \mu \mathrm{L} 70 \% \mathrm{EtOH}$ and 200 
$\mu \mathrm{L}$ of $7.5 \mathrm{M}$ ammonium acetate $\left(\mathrm{AcNH}_{4}\right)$, were added to each microcentrifuge tube and vortexed. The tubes were then placed in a $-20^{\circ} \mathrm{C}$ freezer overnight to precipitate DNA. Once extracted, gDNA was treated twice with a mixture of RNAse A/ RNAse T1 at 10 $\mathrm{ug} / \mu \mathrm{L}$ for $1 \mathrm{~h}$ with phenol extraction and ethanol precipitation steps between the two treatments to ensure the total elimination of RNA.

\section{gDNA Enzymatic Digestion Protocols}

Benzonase. $100 \mathrm{ng}$ of gDNA was dissolved in $100 \mu \mathrm{L}$ of $10 \mathrm{mM}$ Tris- $\mathrm{HCl}$ buffer $\mathrm{pH} 7.9$ containing $10 \mathrm{mM} \mathrm{MgCl} 2,50 \mathrm{mM} \mathrm{NaCl}, 5 \mu \mathrm{M} \mathrm{BHT}$ from Sigma (B1378-100 G), and $3 \mathrm{mM}$ deferoxamine from Sigma (\# BP987). Next, $3 \mathrm{U}$ of benzonase (in $20 \mathrm{mM}$ Tris- $\mathrm{HCl}$ pH 8. 0.2mM MgCl 2 , and $20 \mathrm{mM} \mathrm{NaCl}$ ), $4 \mathrm{mU}$ phosphodiesterase I from Sigma (\# P3243-1VL), $3 \mathrm{U}$ DNAse I, $2 \mathrm{mU}$ of phosphodiesterase II from Sigma (\# P9041-10 UN) and $2 \mathrm{U}$ of alkaline phosphatase from Biolabs (\# M0290) were added and the mixture was incubated at $37^{\circ} \mathrm{C}$. Finally, the mixture was transferred in a microspin filter from Agilent Technologies (\# 5190-5275) and recovered after spinning at $20^{\circ} \mathrm{C}$ for $10 \mathrm{~min}$ at 14000 rpm.

Nuclease P1. We used a modified protocol from (Wang et al; 2011) ${ }^{31} .100 \mathrm{ng}$ of gDNA was dissolved in a $10 \mu \mathrm{L}$ of buffer containing $0.3 \mathrm{M} \mathrm{AcONa} \mathrm{pH} \mathrm{5.6,10} \mathrm{mM} \mathrm{ZnCl} 2,3 \mathrm{~m} \mathrm{M}$ deferoxamine, and 1 mM EHNA. Next, 4 U of Nuclease P1 from Sigma (\# N8630-1VL) (in $30 \mathrm{mM} \mathrm{AcONa} \mathrm{pH} 5.3,5 \mathrm{mM} \mathrm{ZnCl}_{2}$ and $50 \mathrm{mM} \mathrm{NaCl}$ ) and $5 \mathrm{mU}$ phosphodiesterase II from Sigma (\# P9041-10UN) were added and the mixture was incubated at $37^{\circ} \mathrm{C}$. After 4 h, $4 \mathrm{U}$ of alkaline phosphatase from Biolabs (\# M0290) and $5 \mathrm{mU}$ of phosphodiesterase I from Sigma (\# P3243-1VL) were added and the mixture was incubated for additionally 2h. Finally, the mixture was transferred in a microspin filter from Agilent Technologies (\# 5190-5275) and recovered after spinning at $20^{\circ} \mathrm{C}$ for $10 \mathrm{~min}$ at $14000 \mathrm{rpm}$. 
NEB Nucleoside Digestion kit. 100 ng of gDNA was digested according to the Biolabs New England manufacture using the Nucleoside Digestion kit (\# M0649), with little modification on time incubation, extended to overnight at $37^{\circ} \mathrm{C}$. Finally, the mixture was transferred in a microspin filter from Agilent Technologies (\# 5190-5275) and recovered after spinning at $20^{\circ} \mathrm{C}$ for $10 \mathrm{~min}$ at $14000 \mathrm{rpm}$.

DNA Degradase Plus kit. $100 \mathrm{ng}$ of gDNA was digested according to the Zymo Research manufacture using DNA Degradase Plus ${ }^{\mathrm{TM}}$ kit (\# E 2020), with little modification on time incubation, extended to overnight at $37^{\circ} \mathrm{C}$. Finally, the mixture was transferred in a microspin filter $(3 \mathrm{kDa})$ and recovered after spinning at $20^{\circ} \mathrm{C}$ for $10 \mathrm{~min}$ at $14000 \mathrm{rpm}$.

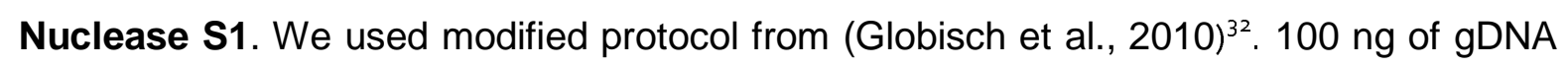
was heated to $100^{\circ} \mathrm{C}$ for 5 min to denature the DNA and rapidly cooled on ice. Buffer $\mathrm{A}$ (10 $\mu \mathrm{L}, 300 \mathrm{mM}$ ammonium acetate, $\left.100 \mu \mathrm{M} \mathrm{CaCl}_{2}, 1 \mathrm{mM} \mathrm{ZnSO}_{4}, \mathrm{pH} 5.7\right)$ and nuclease S1 from Sigma (\# N5661-50 KU) (80 U, Aspergillus oryzae) were added to the mixture and incubated for $3 \mathrm{~h}$ at $37^{\circ} \mathrm{C}$. Addition of buffer $\mathrm{B}(12 \mathrm{~mL}, 500 \mathrm{mM}$ Tris- $\mathrm{HCl}, 1 \mathrm{mM}$ EDTA), Antarctic phosphatase from NEB (\# M0289S-10 units), snake venom phosphodiesterase I (0.2 units, Crotalus adamanteus venom) from Sigma (\# P3243-1VL) and incubation for further $3 \mathrm{~h}$ at $37^{\circ} \mathrm{C}$ completed the digestion. Finally, the mixture was transferred in a microspin filter from Agilent Technologies (\# 5190-5275) and recovered after spinning at $20^{\circ} \mathrm{C}$ for $10 \mathrm{~min}$ at $14000 \mathrm{rpm}$.

\section{Quantification of DNA cytosine modifications by LC-MS/MS}

Analysis of global levels of $5 \mathrm{mdC}, 5 \mathrm{hmdC}, 5 \mathrm{fdC}$ and $5 \mathrm{cadC}$ (herein abbreviated $5 \mathrm{mC}, 5 \mathrm{hmC}, 5 \mathrm{fC}$ and $5 \mathrm{caC}$ ) was performed on a $\mathrm{Q}$ exactive mass spectrometer (Thermo Fisher Scientific). It was equipped with an electrospray ionisation source $(\mathrm{H}-$ 
ESI II Probe) coupled with an Ultimate 3000 RS HPLC (Thermo Fisher Scientific). DNA digestions were injected onto a Thermo Fisher Hypersil Gold aQ chromatography column $(100 \mathrm{~mm} * 2.1 \mathrm{~mm}, 1,9$ um particle size $)$ heated at $30^{\circ} \mathrm{C}$. The flow rate was set at $0.3 \mathrm{~mL} / \mathrm{min}$ and run with an isocratic eluent of $1 \%$ acetonitrile in water with $0.1 \%$ formic acid for 10 minutes. Parent ions were fragmented in positive ion mode with $10 \%$ normalized collision energy in parallelreaction monitoring (PRM) mode. MS2 resolution was 17,500 with an AGC target of $2 \mathrm{e} 5$, a maximum injection time of $50 \mathrm{~ms}$ and an isolation window of $1.0 \mathrm{~m} / \mathrm{z}$. The inclusion list contained the following masses: dC (228.1), 5mdC (242.1), 5hmdC (258.1), 5fdC (256.1) and 5cadC (272.1). Extracted ion chromatograms of base fragments $( \pm 5 p p m)$ were used for detection and quantification (112.0506 Da for $\mathrm{dC}$; 126.0662 Da for $5 \mathrm{mdC} ; 142.0609$ for $5 \mathrm{hmdC} ; 140.0449$ for $5 \mathrm{fdC}$ and 156.0399 for 5 cadC). Calibration curves were previously generated using synthetic standards in the ranges of 0.2 to 50 pmoles injected for $\mathrm{dC}$ and 0.02 to 10 pmoles for $5 \mathrm{mdC}$, 5hmdC, $5 \mathrm{fdC}$ and 5cadC. Results are expressed as a percentage of total $\mathrm{dC}$

\section{Yeast culture and strain construction}

Standard methods were used for cultivation and manipulation of yeast strains ${ }^{33}$. Epitope-tagging was performed as described previously ${ }^{34}$. In order to express Dnmt2 ${ }^{p f}$-SNAP, the coding sequence of the SNAP-tag was PCR amplified using the source plasmid pAct -SNAP-3XGGGs-GW gift from Anne Plessis Lab and the primers GCGGGTACCATGGACAAAGACTGCGAAATG and GCGCTCGAGTCATTAGCCCAGCCCAGGCTTGCCCAG. The 2,713 kbp Hindll Xhol fragment containing the Dnmt2-SNAP fused sequence was excised and inserted into pRS416 plasmid gift from GF Richard lab. This vector is a centromeric 
(i.e., low-copy number) plasmid designed for the expression in yeast of hybrid proteins with SNAP fluorescent protein at the N-terminus.

\section{SNAP immunofluorescence assay}

For the evaluation of yeast transformation, the commercially available fluorescent ligand SNAP-Cell TMR-Star (New England Biolabs) was used.

For image acquisition, an epifluorescence microscope (Leica Microsystems, Germany) equipped with an oil immersion lens (1.4 NA; 100 x; DM200 LED; Leica Microsystems), a N3 filter cube (excitation: 546/12 nm; emission: 600/40 nm) and a GFP filter cube (excitation: 450/90 nm; emission: LP $590 \mathrm{~nm}$ ) was employed.

\section{Dot Blot Assay}

$20 \mu \mathrm{g}$ of gDNA was denatured at $100^{\circ} \mathrm{C}$ for $10 \mathrm{~min}$. Samples were rapidly chilled for 5 minutes on wet ice and then applied to a positively charged nylon membrane under vacuum using a 96-well Dot Blot Hybridization Manifold (Harvard Apparatus Limited, Holliston, MA, USA). The membrane was washed twice in $2 \times$ SSC buffer and UVcrosslinked. The membrane was probed with anti- bodies specific to $5 \mathrm{mC}$ (Active motif \# 39649; dilution factor 1:1. 000). To control loading, the membrane was probed with a rabbit poly-clonal antibody raised against single-stranded DNA (Demeditec Diagnostics, Kiel, Schleswig-Holstein, Germany). Following treatment with enhanced chemiluminescence substrate, membranes were scanned on an Image Quant LAS 4000 (GE Healthcare, Little Chalfont, Buckinghamshire, UK) imaging station. 


\section{DNA content quantification by flow cytometry}

Parasites cultured at $5 \%$ or $20 \% \mathrm{O}_{2}$ were fixed in $4 \%$ PFA $+0,0075 \%$ glutaraldehyde in 1x PBS. Fixed cells were then stained with 2X SYBR-Green and the mean fluorescence intensity was measured using a BD Fortessa FACS instrument and analyzed using FlowJo software.

\section{Statistics}

Statistical analysis was carried out a 2-way ANOVA significance test, unless otherwise specified.

\section{Results}

\section{An optimized mass spectrometry-based protocol for the detection of DNA cytosine modifications in AT-rich gDNA}

In order to accurately investigate the levels of DNA cytosine epigenetic marks in the highly AT-rich genomic DNA (gDNA) of $P$. falciparum using LC-MS/MS, we compared the gDNA digestion and hydrolysis protocol using five different methods: Nuclease P1, Benzonase, NEB Nucleoside Digestion kit, Degradase Plus kit and Nuclease S1 (Figure1). All methods showed similar levels of $5 \mathrm{mC}$ and oxidative cytosine forms in gDNAs from mouse Embryonic Stem Cells (mESC) (Figure1A). However, in the case of malaria parasites, the detected levels of $5 \mathrm{hmC}$ varied dramatically dependent on the method used to digest and hydrolyze gDNA. The levels of 5hmC in Plasmodium gDNA samples treated with Nuclease P1 are up to 8-fold higher compared to all other hydrolysis methods (Figure1B and Figure S1). Thus, our data show that Nuclease P1 (NP1) digestion is by far the most efficient enzyme to detect $5 \mathrm{hmC}$ in an extremely AT- 
rich organism. Therefore, the NP1 hydrolysis method was adopted to explore cytosine methylation marks in $P$. falciparum.

\section{DNA cytosine modifications are dynamically regulated during the 48-hour blood stage cycle of $P$. falciparum}

Parasites were synchronized and harvested at the ring (10-15 hours post invasion; hpi), trophozoite (24-30hpi) and schizont stages (35-40hpi) to determine how the levels of $5 \mathrm{mC}$ and its oxidative derivatives vary across the intraerythrocytic developmental cycle (IDC) of $P$. falciparum. Using the LC-MS/MS protocol on DNA digested with Nuclease P1, we show that the levels of DNA cytosine modifications in malaria parasites dynamically change as the parasite transitions from the early ring stage to the mature schizonts in the infected red blood cells (iRBCs) (Figure $2 \mathrm{~A}$ ). While $5 \mathrm{mC}$ levels are very low in the ring $(0.077 \% \pm 0.015 \%)$ and schizont stages $(0.13 \% \pm 0.064 \%)$, we observe a drastic increase $(10$-fold $)$ from the ring stage transition to trophozoites $(0.727 \% \pm 0.265 \%)$ (Figure 2A). Surprisingly, $5 \mathrm{hmC}$ levels exhibit the opposite pattern. $5 \mathrm{hmC}$ is by far the predominant cytosine modification in rings $(0.533 \% \pm 0.352 \%)$ and schizonts $(0.697 \%$ $\pm 0.072 \%)$ and decreases by 1.6 -fold at the trophozoite stage $(0.333 \% \pm 0.162 \%)$. In addition, the levels of $5 \mathrm{fC}$ and $5 \mathrm{caC}$ are extremely low in all three stages of the IDC with $5 \mathrm{caC}$ being only detectable in the schizont stages $(0.03 \% \pm 0.01 \%)$.

Along with LC-MS/MS, we used a validated in vitro enzymatic assay ${ }^{35}$ in order to quantify DNA methylation activity (DNMT) in nuclear extracts prepared from the three stages of the IDC. Human recombinant DNMT3aC is used as internal positive control and the data is presented as relative fluorescence units (RFU) (sample)-RFU (background signal) (Figure 2B). Our results show that DNA methylase activity is very low in ring stages (5000 
\pm 200 RFU) with a sharp increase in activity (8-fold) in trophozoites (40000 \pm 200 RFU).

The high activity signal is maintained in schizonts (Figure 2B). The DNMT activity assay data corroborates our LC-MS/MS findings showing the sharp increase of DNA methylation in the trophozoite stages of $P$. falciparum. Altogether, our data shows that DNA cytosine methylation is very dynamic in malaria blood stages and suggests a tight developmental regulation of $5 \mathrm{mC}$ and the oxidative forms throughout $P$. falciparum IDC.

\section{Variable oxygen levels modulate the DNA cytosine methylation epigenetic landscape in $P$. falciparum}

We next wanted to evaluate how different physiological oxygen environments might affect the levels of DNA cytosine modifications in the parasites asexual blood stages. To this end, we analyzed the hyperoxic (atmospheric) $20 \% \mathrm{O}_{2}$ condition mimicking the levels of oxygen that the parasite encounters during Anopheles mosquito vector development. As control, the normoxic $5 \% \mathrm{O}_{2}$ applied in standard $P$. falciparum in vitro cultures was used. Synchronous ring stage parasites were exposed to both $5 \%$ or $20 \% \mathrm{O}_{2}$ levels and trophozoites, schizonts and rings were harvested for each condition during the next 72 hours. Giemsa-stained blood smears showed no morphological differences between trophozoites, schizonts and rings parasites cultured in normoxic or atmospheric $\mathrm{O}_{2}$ conditions (Figure S2). In addition, SYBR green-based DNA quantification showed that $\mathrm{O}_{2}$ levels do not impact parasite DNA content of blood stages (Figure S2) as suggested by previous studies ${ }^{25}$.

Importantly, LC-MS/MS-based DNA modifications quantification shows a shift of $5 \mathrm{mC}$ towards its oxidative forms in parasites cultured under hyperoxic $20 \% \mathrm{O}_{2}$ levels (Figure 3). Notably, exposure of the parasites to hyperoxic $\mathrm{O}_{2}$ levels led to a significant increase in the abundance of $5 \mathrm{caC}$ in all three stages of the IDC (rings, trophozoites and 
schizonts) (Figure 3A, B and C) indicating an increased oxidation of 5hmC. The shift in the DNA methylation/oxidation balance is very clear at the trophozoite stage. $5 \mathrm{mC}$ levels are significantly decreased (14-fold) from $(0.94 \% \pm 0.11 \%)$ in parasites cultured under the control $5 \% \mathrm{O}_{2}$ levels to $(0.065 \% \pm 0.017 \%)$ in those under $20 \% \mathrm{O}_{2}$. The decrease of $5 \mathrm{mC}$ is accompanied by an increase in $5 \mathrm{hmC}(2.5$-fold $)$ from $(0.3 \% \pm 0.1 \%)$ to $(0.74 \% \pm$ $0.04 \%)$, along with the significant increase in $5 \mathrm{caC}(16.5$-fold $)$ from $(0.01 \%)$ to $(0.17 \pm$ $0.07 \%$ ) (Figure 3B). Altogether, the LC-MS/MS data strongly support a direct influence of oxygen $\mathrm{O}_{2}$ concentration on the ratio of $5 \mathrm{mC}$ and its oxidative derivatives, which may allow the malaria parasite to adapt its epigenetic cytosine landscape to variable host environments.

\section{DNA cytosine methylation in $P$. falciparum is determined by DNMT2}

Given that $P$. falciparum belongs to a small group of DNMT2-only organisms ${ }^{27,36}$, we investigated if the loss of Pf-DNMT2 impacts the DNA cytosine methylation repertoire of the parasite. To this end, we used trophozoites genomic DNA from two Pf-DNMT2KO clones (cl1 and $\mathrm{cl} 2$ ) that we generated previously ${ }^{28}$. The LC-MS/MS analysis show that the levels of $5 \mathrm{mC}$ are drastically decreased to background levels in the Pf-DNMT2KO clones $(0.03 \%)$ when compared to the 3D7 control strain $(0.620 \% \pm 0.269 \%)$ (Figure $4 \mathrm{~B})$. Importantly, 5hmC levels were also dramatically decreased in the two Pf-DNMT2KO clones $(0.009 \%)$ when compared to their $3 D 7$ counterparts $(0.42 \% \pm 0.085 \%)$. In addition, loss of $5 \mathrm{mC}$ and $5 \mathrm{hmC}$ was accompanied by a 20 -fold decrease of $5 \mathrm{caC}$ in PfDNMT2KO-cl1 and Pf-DNMT2KO-cl2 (Figure 4B trophozoite and Figure S3 for rings and schizont stage).

To confirm the function of Pf-DNMT2 as a DNA methyltransferase we expressed the fulllength protein in the budding yeast Saccharomyces cerevisiae ( $S$. Cerevisiae), which 
lacks any of the known DNMTs ${ }^{37}$ (Figure $4 \mathrm{C}$ ). The $5 \mathrm{mC}$ content in transformed and nontransformed yeast cells was first assessed using a monoclonal antibody specific to $5 \mathrm{mC}$. Methylated DNA is detectable only in Pf-DNMT2 transformed yeast cells (figure 4D). LCMS/MS analysis of digested DNA isolated from non-transformed yeasts shows no detectable $5 \mathrm{mC}$ as expected. However, in Pf-DNMT2 transformed yeast cells the levels of $5 \mathrm{mC}$ increased up to $0.06 \%$ of total cytosines (Figure $4 \mathrm{E}$ ). Taken together, our data clearly show that Pf-DNMT2 (PF3D7_0727300) is an active DNA methyltransferase responsible of cytosine methylation in the gDNA of $P$. falciparum (Figure 4A). Importantly, the LC-MS/MS data also shows that the presence of $5 \mathrm{hmC}, 5 \mathrm{fC}$ and $5 \mathrm{caC}$ in $P$. falciparum is completely dependent on $5 \mathrm{mC}$ as previously shown for other model systems $^{14,15,20,38}$. In addition, our results infer the presence of a yet unknown TET-like enzyme in $P$. falciparum that triggers the oxidation of $5 \mathrm{mC}$ into $5 \mathrm{hmC}$ and further into $5 \mathrm{fC}$ and $5 \mathrm{caC}$ (Figure 4A).

\section{Discussion}

$P$. falciparum is among the most AT-rich genomes known to date with an overall $A+T$ composition of $80.6 \%$, which rises to $\sim 90 \%$ in introns and intergenic regions ${ }^{39}$. Given this unusual base composition, we compared different nuclease digestion procedures into single nucleosides prior LC-MS/MS analysis of cytosine modifications in genomic DNA. We observed striking differences in the digestion efficacy of $5 \mathrm{hmdC}$ between the distinct nucleases. With Nuclease P1 (NP1) 5hmdC levels are up to 8-fold higher compared to all others digestion methods, highlighting an important difference between AT-rich genomes and organisms with a balanced nucleotide composition. AT-rich DNA sequences display features that could account for the observed low efficacy of digestion of the oxidized DNA cytosine modifications. For example, AT- 
rich DNA stretches can form local curvature ${ }^{40}$ that may exert an influence on the interaction of nucleases with DNA. Furthermore, 5hmC was shown to enhance DNA flexibility $^{41}$, a feature that could change the affinity of certain type of nucleases for 5hmdC containing DNA.

We thus used nuclease P1 for digestion of gDNA to investigate the plasmodial DNA cytosine modifications landscape during the blood stage development using LCMS/MS. Our results show several features not observed previously in other organisms. The ratio between $5 \mathrm{mC}$ and the three oxidized cytosine forms is rather unusual when compared to higher eukaryotes (Figure 1A/B). Furthermore, we observe dynamic changes of $5 \mathrm{mC}$ levels during the 48-hours blood stage parasite cycle. For most of the time, we detect only very low levels of $5 \mathrm{mC}(0.07-0.1 \%)$ and high levels of $5 \mathrm{hmC}(0.5-0.9 \%)$. But during the short period of parasite proliferation (trophozoite stage), the $5 \mathrm{mC} / 5 \mathrm{hmC}$ ratio is inverted (Figure $2 \mathrm{~A}$ ). The reason for the observed dynamic changes remains elusive. While ring stages circulate in the blood, trophozoite stages start to be sequestered in capillaries of host organs. It is tempting to speculate that the change in parasite tropism during blood stage development may be linked to increased DNA methyltransferase activity or reduced DNA demethylation activity. Further studies are necessary to explore the underlying molecular basis. The levels of $5 \mathrm{fC}$ and $5 \mathrm{caC}$ remain very low in all three stages (standard in vitro culture condition), a situation similar to what has been reported in other eukaryotes.

Previous work has reported relatively low $5 \mathrm{mC}$ levels in $P$. falciparum genomic DNA $^{23,24}$. In addition, LC-MS/MS analysis did not detect $5 \mathrm{hmC}$ but pointed to a cytosine base modification with unknown biochemical identity ${ }^{23}$. The digestion protocol used in this report was based on the benzonase nuclease. It is important to note that the unusual base reported earlier was also detectable in our nuclease 
digestion protocols (Figure S1). We observed that this cytosine form was predominant in $P$. falciparum gDNA hydrolyzed with benzonase but present only at very low levels when the DNA was digested with Nuclease P1 (Figure S1). We conclude that the choice of nuclease for the digestion of AT-rich DNA is important to maximize the liberation of oxidative cytosine nucleosides.

Experimental evidence from the cancer field demonstrated that reduction of oxygen levels in tumors is associated with a reduced ability to produce $5 \mathrm{hmC}^{22}$. Since $P$. falciparum is exposed to fluctuating oxygen concentrations during the life cycle, we investigated if malaria parasite cytosine modification marks respond to physiological changes in oxygen levels. Our results demonstrate that host-dependent oxygen concentrations result in important alterations in the cytosine modification landscape of $P$. falciparum. We observed a shift towards oxidative derivatives of $5 \mathrm{mC}$ at higher oxygen levels (Figure 3). DNA cytosine modifications are now considered to function as distinct epigenetic marks with distinct profiles in tissues/organs in higher eukaryotes $^{38}$. A recent study used anti $5 \mathrm{hmC}$ antibodies to immunoprecipitate DNA regions enriched for this cytosine mark (hmeDIP-seq) of gDNA of $P$. falciparum. The presence of hydroxymethylated cytosine in gene bodies positively correlated with transcript levels in blood stages ${ }^{23}$. We hypothesize that the observed dynamic changes of the cytosine modifications may contribute to the malaria pathogen adaptation to changing environments, in particular during $P$. falciparum development within the mosquito vector.

TET enzymes, which catalyze the oxidative cytosine forms depend on oxygen as cosubstrate $^{15,21}$, may sense changes in oxygen levels and change the basal cytosine modification level toward oxidative cytosine forms. For example, TET1 activity as well as $5 \mathrm{hmC}$ levels are decreased in low oxygen conditions leading to the inhibition of 
the differentiation of mouse embryonic stem cells (mESC) ${ }^{42}$. Although TET-like activity was previously detected in nuclear extracts of $P$. falciparum using a TET hydroxylase activity kit (Abcam) no obvious ortholog of mammalian TET enzymes could be identified to date ${ }^{23}$. Further studies are needed to identify TET-like enzymes in malaria parasites and to explore the role of the oxidized cytosines as potential epigenetic marks.

An obvious question is if the identified oxidative cytosine forms (5hmC, $5 \mathrm{fC}, 5 \mathrm{caC}$ ) depend on $5 \mathrm{mC}$ as a precursor or are formed by oxidative lesions. $P$. falciparum is part of a small number of organisms called DNMT2-only, given the absence of genes coding for the canonical DNA methyltransferase genes DNMT1 and DNMT3 ${ }^{36}$. A recent study reported a $P$. falciparum Pf-DNMT2 KO parasite line, and this work confirmed the well-established function of DNMT2 as tRNA aspartic acid (GTC) cytosine methyltransferase ${ }^{28}$. These $\mathrm{KO}$ parasites show no growth defect but are significantly more sensitive to various stressor and respond to specific stress situations by 8-fold higher transmission stages or higher drug sensitivity, respectively. This study also observed a reduction of $5 \mathrm{mC}$ in gDNA of $\mathrm{KO}$ parasites, however, given the very low level reported for $5 \mathrm{mC}(0.06 \%)$ in the investigated schizont stage, it remained unclear if the observed reduction in $5 \mathrm{mC}$ can be attributed to the loss of Pf-DNMT2 or variations in the experimental procedure ${ }^{28}$. DNMT2-dependent DNA methylation is still disputed with contradictory results from distinct DNMT2-only organisms $^{27}$. Here we demonstrate that DNMT2 has a dual function using tRNA and DNA as substrate in malaria parasites. Quantitative LC-MS/MS analysis demonstrate that the levels of $5 \mathrm{mC}$ are drastically decreased to background levels in the PfDNMT2KO clones $(0.03 \%)$ when compared to the 3D7 control strain $(0.62 \%)$ (Figure 4B). Furthermore, the identified oxidative cytosine forms are also reduced to 
background levels, inferring that $5 \mathrm{mC}$ is the precursor for $5 \mathrm{hmC}, 5 \mathrm{fC}$ and $5 \mathrm{caC}$ cytosine modifications.

In conclusion, this work demonstrates that plasmodial Pf-DNMT2 has a dual function in methylating tRNA and DNA. The $5 \mathrm{mC}$ methylation of gDNA is developmentally regulated during the blood stage development and the $5 \mathrm{mC}$ distribution versus oxidized forms can vary dramatically depending on environmental oxygen concentrations. Expanding the number of potential epigenetic DNA marks in this pathogen may open new avenues to understand the regulatory circuits that act during the life cycle stage.

\section{Acknowledgements}

This work was supported by the Laboratoire d'Excellence (LabEx) ParaFrap [ANR11-LABX-0024], the Agence Nationale pour la Recherche [Project EpiKillMal, ANR20-CE18-0006]. This work was supported by DIM1Health 2019 equipment grant "EpiK” from Région lle de France.

\section{Author contributions}

Conceptualization A.S, E.H, S.M and B.A; Methodology: E.H, S.M and F.B;

Investigation E.H and S.M; Analysis: E.H, S.M and F.B; Writing: E.H, S.M and A.S; Funding acquisition: A.S and P.B.

\section{Data availability}

LC-MS/MS raw data is shown in supplementary tables S1 and S2. 


\section{References}

1. Hotchkiss, R. D. The quantitative separation of purines, pyrimidines, and nucleosides by paper chromatography. J. Biol. Chem. 175, 315-332 (1948).

2. Holliday, R. \& Pugh, J. E. DNA modification mechanisms and gene activity during development. Science 187, 226-232 (1975).

3. Riggs, A. D. X inactivation, differentiation, and DNA methylation. Cytogenetic and Genome Research 14, 9-25 (1975).

4. Aapola, U. et al. Isolation and initial characterization of a novel zinc finger gene, DNMT3L, on 21q22.3, related to the cytosine-5-methyltransferase 3 gene family. Genomics 65, 293-298 (2000).

5. Bachman, K. E., Rountree, M. R. \& Baylin, S. B. Dnmt3a and Dnmt3b Are Transcriptional Repressors That Exhibit Unique Localization Properties to Heterochromatin. Journal of Biological Chemistry 276, 32282-32287 (2001).

6. Baubec, T. et al. Genomic profiling of DNA methyltransferases reveals a role for DNMT3B in genic methylation. Nature 520, 243-247 (2015).

7. Sharif, J. et al. The SRA protein Np95 mediates epigenetic inheritance by recruiting Dnmt1 to methylated DNA. Nature 450, 908-912 (2007).

8. Doi, A. et al. Differential methylation of tissue- and cancer-specific CpG island shores distinguishes human induced pluripotent stem cells, embryonic stem cells and fibroblasts. Nat. Genet. 41, 1350-1353 (2009). 
9. Jones, P. A. Functions of DNA methylation: islands, start sites, gene bodies and beyond. Nat. Rev. Genet. 13, 484-492 (2012).

10. Guy, J., Cheval, H., Selfridge, J. \& Bird, A. The Role of MeCP2 in the Brain. Annual Review of Cell and Developmental Biology 27, 631-652 (2011).

11. Meng, H. et al. DNA Methylation, Its Mediators and Genome Integrity. International Journal of Biological Sciences 11, 604-617 (2015).

12. Bienvenu, T. \& Chelly, J. Molecular genetics of Rett syndrome: when DNA methylation goes unrecognized. Nature Reviews Genetics 7, 415-426 (2006).

13. Shi, D.-Q., Ali, I., Tang, J. \& Yang, W.-C. New Insights into 5hmC DNA Modification: Generation, Distribution and Function. Front Genet 8, 100 (2017).

14. Wu, X. \& Zhang, Y. TET-mediated active DNA demethylation: mechanism, function and beyond. Nat. Rev. Genet. 18, 517-534 (2017).

15. Ito, S. et al. Tet Proteins Can Convert 5-Methylcytosine to 5-Formylcytosine and 5-Carboxylcytosine. Science 333, 1300-1303 (2011).

16. Mellén, M., Ayata, P., Dewell, S., Kriaucionis, S. \& Heintz, N. MeCP2 Binds to 5hmC Enriched within Active Genes and Accessible Chromatin in the Nervous System. Cell 151, 1417-1430 (2012).

17. Sun, W., Zang, L., Shu, Q. \& Li, X. From development to diseases: The role of 5hmC in brain. Genomics 104, 347-351 (2014).

18. Neri, F. et al. Single-Base Resolution Analysis of 5-Formyl and 5-Carboxyl Cytosine Reveals Promoter DNA Methylation Dynamics. Cell Reports 10, 674683 (2015).

19. Song, C.-X. \& He, C. Potential functional roles of DNA demethylation intermediates. Trends in Biochemical Sciences 38, 480-484 (2013). 
20. Tahiliani, M. et al. Conversion of 5-methylcytosine to 5-hydroxymethylcytosine in mammalian DNA by MLL partner TET1. Science 324, 930-935 (2009).

21. Matuleviciute, R., Cunha, P. P., Johnson, R. S. \& Foskolou, I. P. Oxygen regulation of TET enzymes. FEBS J febs.15695 (2021) doi:10.1111/febs.15695.

22. Thienpont, B. et al. Tumour hypoxia causes DNA hypermethylation by reducing TET activity. Nature 537, 63-68 (2016).

23. Hammam, E. et al. Discovery of a new predominant cytosine DNA modification that is linked to gene expression in malaria parasites. Nucleic Acids Research 48, 184-199 (2020).

24. Ponts, N. et al. Genome-wide Mapping of DNA Methylation in the Human Malaria Parasite Plasmodium falciparum. Cell Host \& Microbe 14, 696-706 (2013).

25. Archer, N. M. et al. Resistance to Plasmodium falciparum in sickle cell trait erythrocytes is driven by oxygen-dependent growth inhibition. Proc Natl Acad Sci USA 115, 7350-7355 (2018).

26. Ha, Y.-R., Ryu, J., Yeom, E. \& Lee, S.-J. Comparison of the tracheal systems of Anopheles sinensis and Aedes togoi larvae using synchrotron X-ray microscopic computed tomography (respiratory system of mosquito larvae using SR- $\mathrm{CCT}$ ): HA ET AL . Microsc Res Tech 80, 985-993 (2017).

27. Jeltsch, A. et al. Mechanism and biological role of Dnmt2 in Nucleic Acid Methylation. RNA Biol 14, 1108-1123 (2017).

28. Hammam, E. et al. Malaria Parasite Stress Tolerance Is Regulated by DNMT2Mediated tRNA Cytosine Methylation. mBio e02558-21 (2021) doi:10.1128/mBio.02558-21.

29. Trager, W. \& Jensen, J. Human malaria parasites in continuous culture. Science 193, 673-675 (1976). 
30. Sambi, M. et al. Acellular Mouse Kidney ECM can be Used as a Three-

Dimensional Substrate to Test the Differentiation Potential of Embryonic Stem

Cell Derived Renal Progenitors. Stem Cell Rev and Rep 13, 513-531 (2017).

31. Wang, J. et al. Quantification of Oxidative DNA Lesions in Tissues of Long-Evans

Cinnamon Rats by Capillary High-Performance Liquid Chromatography-Tandem

Mass Spectrometry Coupled with Stable Isotope-Dilution Method. Anal. Chem.

83, 2201-2209 (2011).

32. Globisch, D. et al. Tissue Distribution of 5-Hydroxymethylcytosine and Search for Active Demethylation Intermediates. PLOS ONE 5, e15367 (2010).

33. Sherman, F. Getting started with yeast. in Methods in Enzymology vol. 350 3-41 (Elsevier, 2002).

34. Andresen, M., Schmitz-Salue, R. \& Jakobs, S. Short Tetracysteine Tags to $\beta$ Tubulin Demonstrate the Significance of Small Labels for Live Cell Imaging. MBoC 15, 5616-5622 (2004).

35. Ceccaldi, A. et al. C5-DNA Methyltransferase Inhibitors: From Screening to Effects on Zebrafish Embryo Development. ChemBioChem 12, 1337-1345 (2011).

36. Raddatz, G. et al. Dnmt2-dependent methylomes lack defined DNA methylation patterns. Proc. Natl. Acad. Sci. U.S.A. 110, 8627-8631 (2013).

37. Capuano, F., Mülleder, M., Kok, R., Blom, H. J. \& Ralser, M. Cytosine DNA methylation is found in Drosophila melanogaster but absent in Saccharomyces cerevisiae, Schizosaccharomyces pombe, and other yeast species. Anal. Chem. 86, 3697-3702 (2014). 
38. Carell, T., Kurz, M. Q., Müller, M., Rossa, M. \& Spada, F. Non-canonical Bases in the Genome: The Regulatory Information Layer in DNA. Angew. Chem. Int. Ed. 57, 4296-4312 (2018).

39. Gardner, M. J. et al. Genome sequence of the human malaria parasite Plasmodium falciparum. Nature 419, 498-511 (2002).

40. Hagerman, P. J. Sequence dependence of the curvature of DNA: a test of the phasing hypothesis. Biochemistry 24, 7033-7037 (1985).

41. Ngo, T. T. M. et al. Effects of cytosine modifications on DNA flexibility and nucleosome mechanical stability. Nat Commun 7, 10813 (2016).

42. Burr, S. et al. Oxygen gradients can determine epigenetic asymmetry and cellular differentiation via differential regulation of Tet activity in embryonic stem cells. Nucleic Acids Research 46, 1210-1226 (2018).

\section{Figures and figures legends}


A.

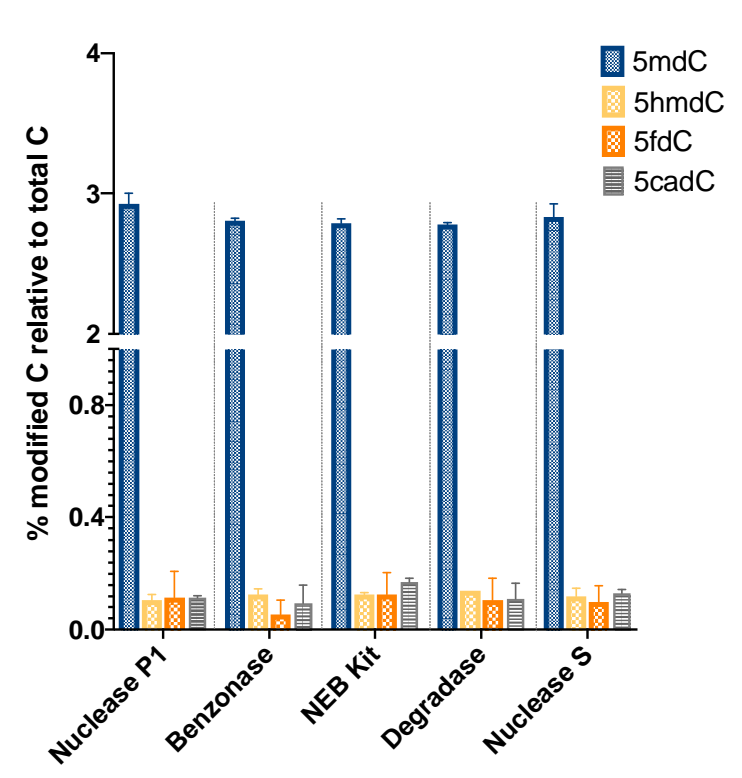

B.

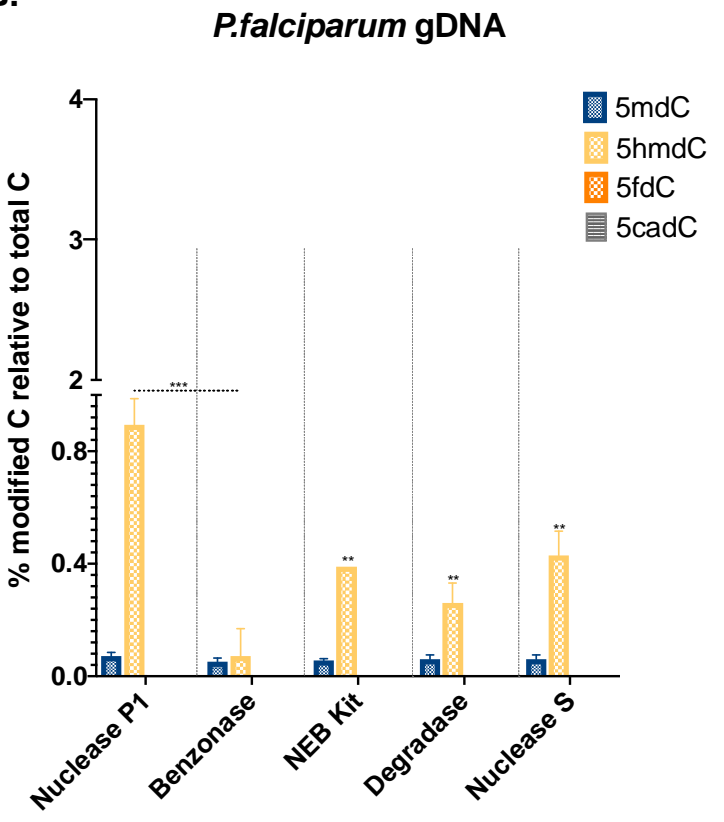

Figure 1: Comparative cytosine modification analysis by LC-MS/MS of gDNA from $P$. falciparum and mouse embryonic stem cells (mESC) using different digestion methods.

Genomic DNA from mESC cells $(A)$ and $P$. falciparum (B) was digested using five different protocols: Nuclease P1(NP1), Benzonase, NEB Nucleoside Digestion kit (NEB kit), Degradase kit (Degradase) and Nuclease S. Quantitative data analysis is shown as percentage of modified deoxycytidines relative to the total number of deoxycytidines in the tested sample and represents the mean ( \pm SD) of two independent replicates for each condition $(n=2)$. Statistical analysis was carried out using a 2way ANOVA test. P Value (Benzonase/NP1) $=0,0007\left({ }^{* * *}\right) ; \mathrm{P}$ Value (NEB kit/NP1) $=0,0048\left(^{* *}\right) ; P$ Value (Degradase/NP1) $=0,002^{(* *}$ ) and P Value (Nuclease $\mathrm{S} / \mathrm{NP} 1)=0,0065\left({ }^{* *}\right)$. 
A.

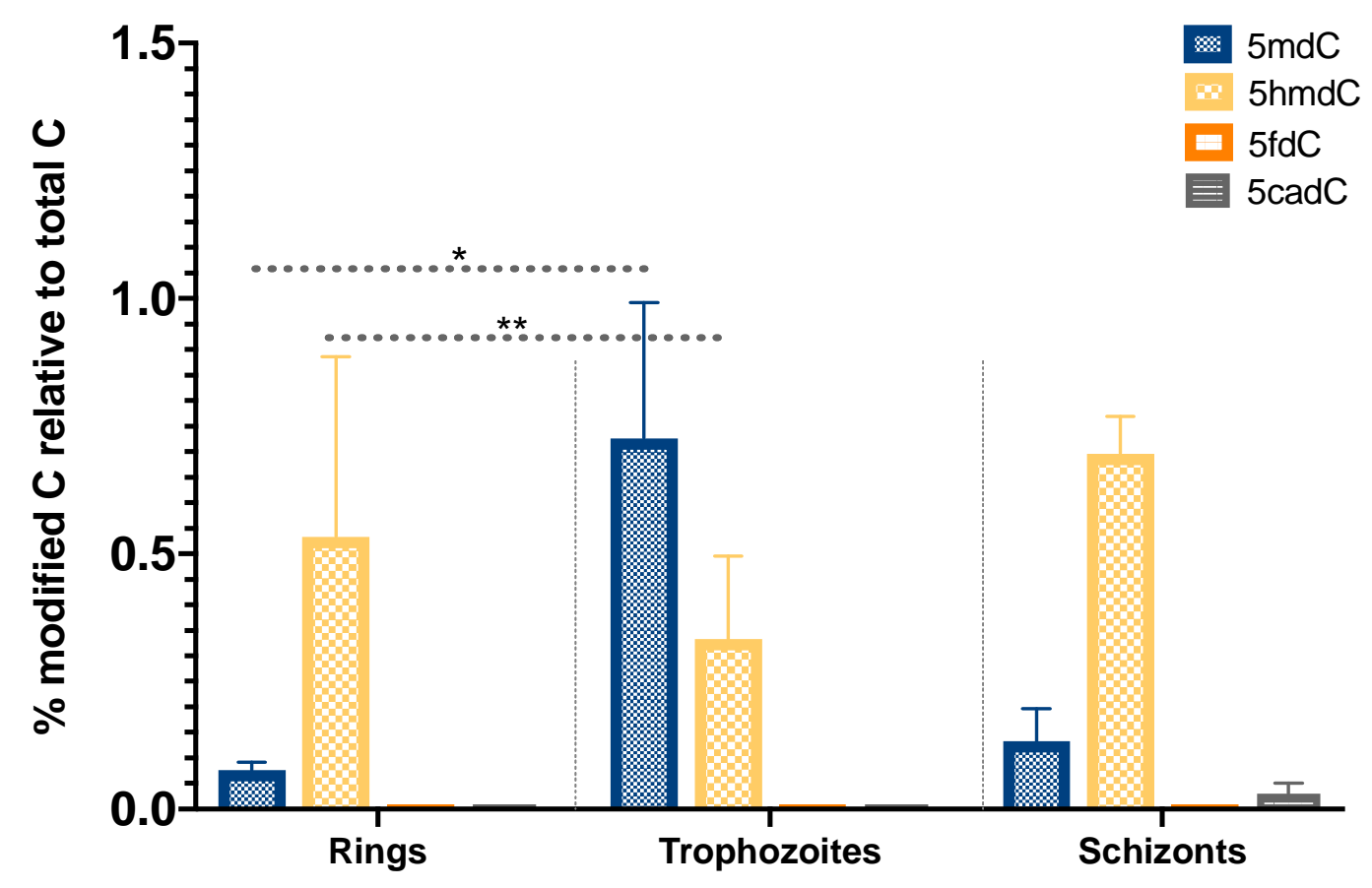

B.

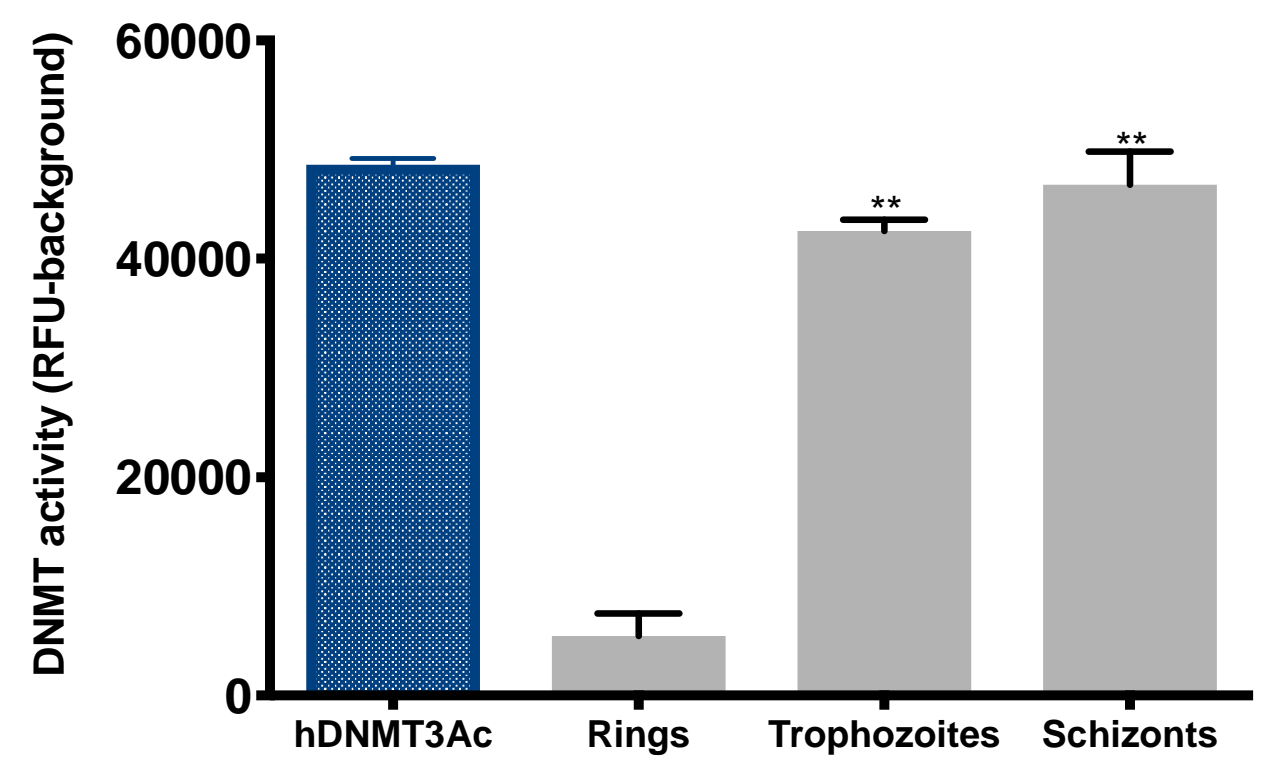

Figure 2: DNA cytosine methylation is dynamically regulated during the 48hour $\boldsymbol{P}$. falciparum asexual blood stage development. 
A. LC-MS/MS-based quantification of $5 \mathrm{mdC}, 5 \mathrm{hmdC}, 5 \mathrm{fdC}$ and $5 \mathrm{cadC}$ in gDNA of synchronized ring, trophozoite and schizont stages. Data is shown as percentage of modified nucleoside relative to the total number of deoxycytidines in each sample and represents the mean of three independent replicates for each stage $(n=3)$.

B. DNA methyltransferase (DNMT) activity assay using nuclear extracts from synchronous rings, trophozoite and schizonts. Data is shown as the RFU (sample)RFU (background) and represents the mean $( \pm S D$ ) of two independent replicates for each stage $(n=2)$. Human recombinant DNMT3aC was used as internal positive control.

Statistical analysis was carried out using a 2way ANOVA for the LC-MS/MS data and a t-test for the DNMT activity data. P Value $<0.05={ }^{*} ; \mathrm{P}$ Value $<0.005={ }^{* *}$ 
A.

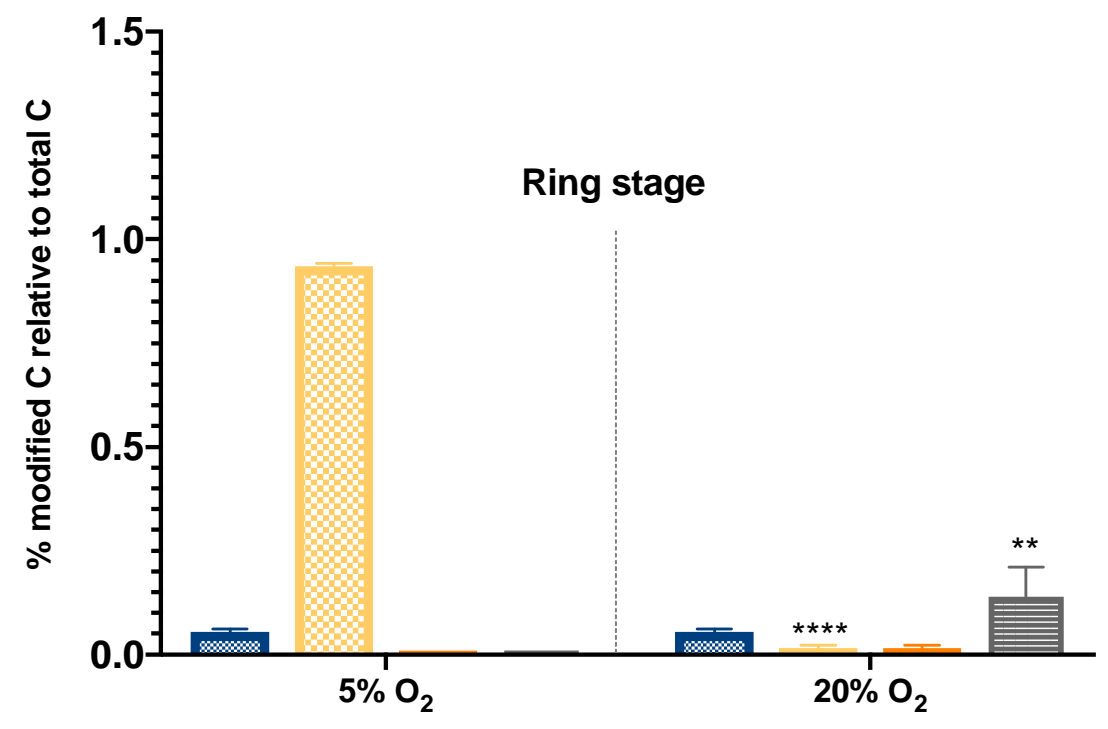

B.

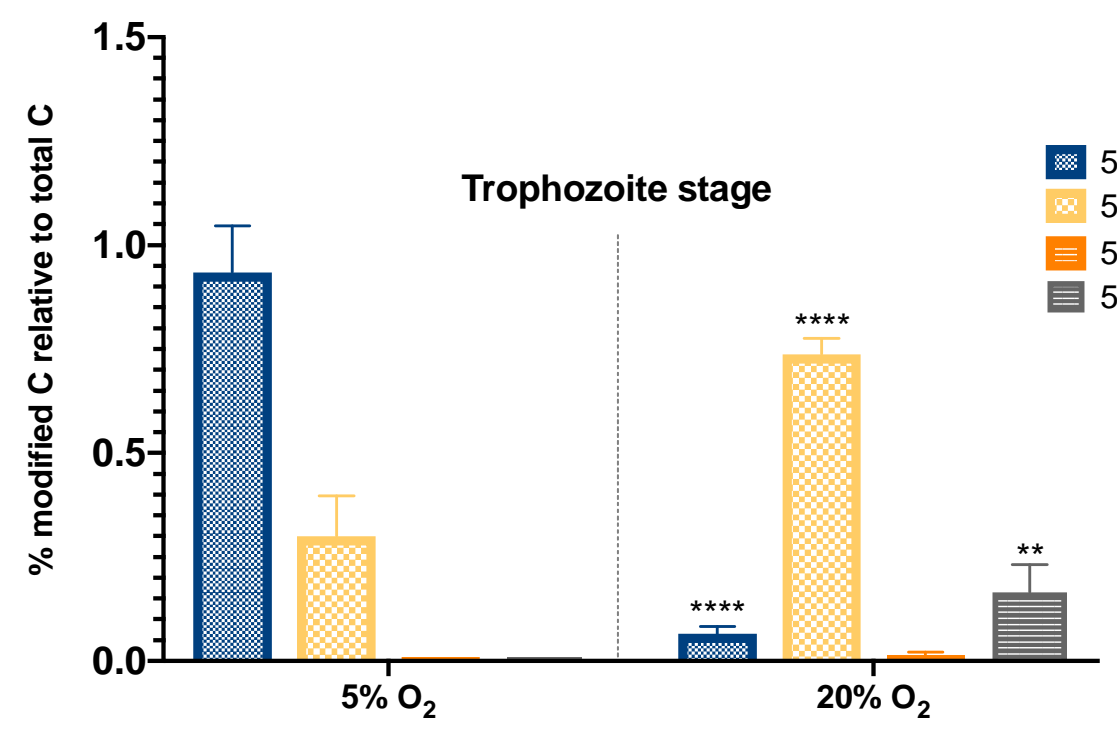

C.

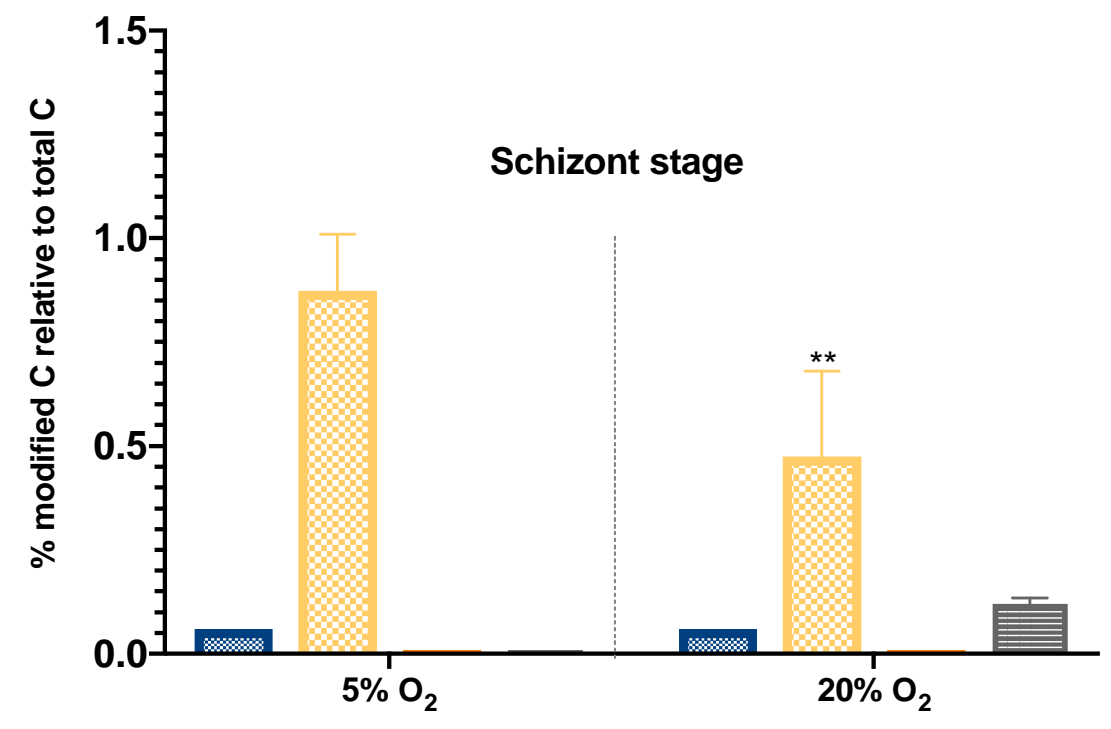




\section{Figure 3: Effect of variable oxygen concentrations on gDNA cytosine} modifications.

DNA was isolated from in vitro cultured parasites cultured at different oxygen levels.

Data is shown as percentage of modified deoxycytidines relative to the total number of deoxycytidines in each sample and represents the mean ( \pm SD) of two independent replicates rings $(A)$ and schizonts $(C)(n=2)$ and four independent replicates for the trophozoite $(B)(n=4)$. Statistical analysis was carried out using a 2way ANOVA test. P Value $<0.0001={ }^{* * *} ; \mathrm{P}$ Value $<0.005=^{* *}$ 
A.

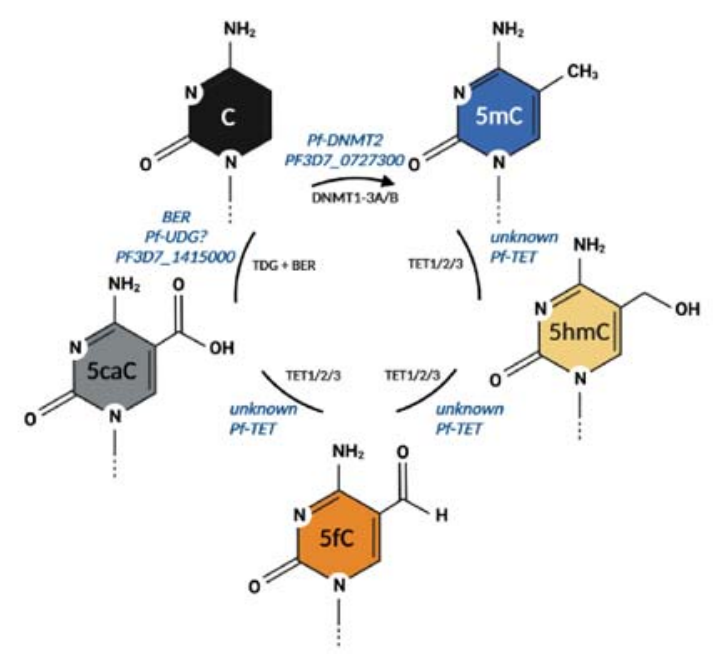

c.
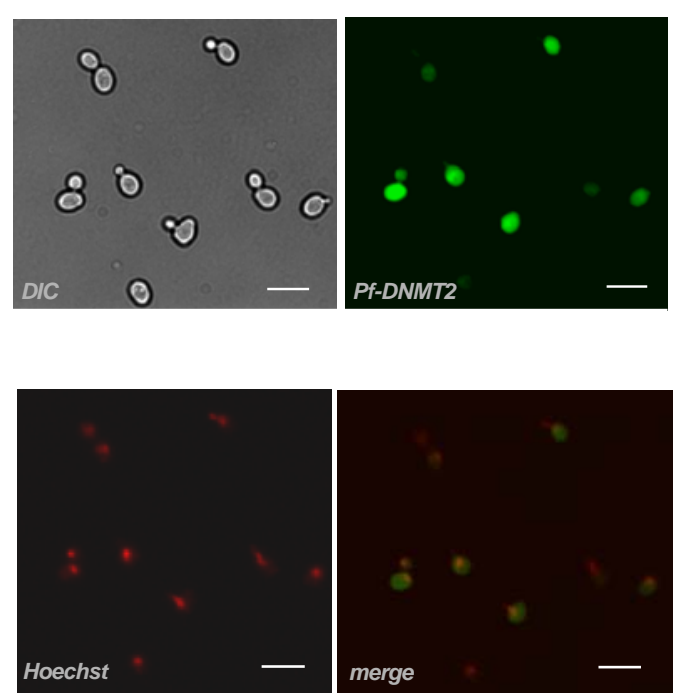

B.

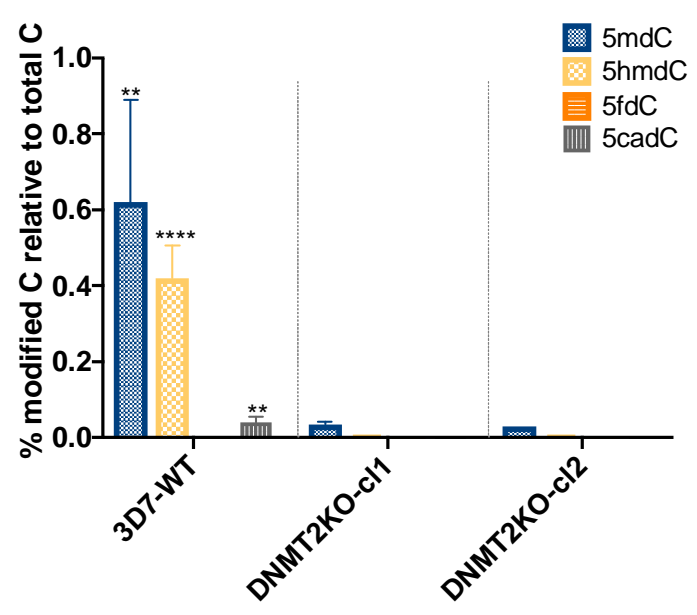

D.

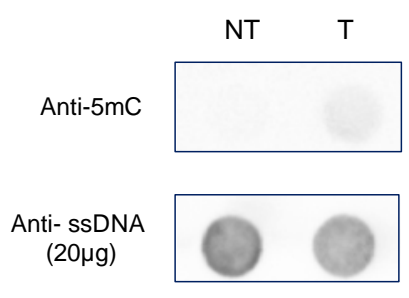

E.

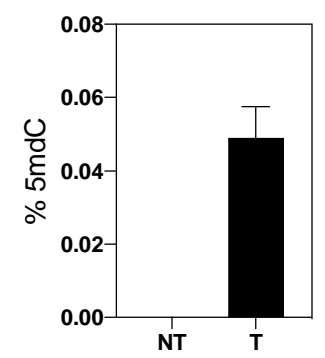

Figure 4: DNA cytosine methylation marks in $P$. falciparum are dependent on the activity of Pf-DNMT2.

A. Schematic representation summarizing the DNA methylation/demethylation pathway as established in model organisms (black font) compared to what is known about the DNA methylation/demethylation machinery in $P$. falciparum (blue font). Pf- 
DNMT2: $P$. falciparum DNA methyltransferase 2; TET: ten eleven translocation enzymes; BER: base excision repair machinery; TGD/UDG: thymine/uracil-DNA glycosylase.

B. LC-MS/MS-based quantification of $5 \mathrm{mdC}, 5 \mathrm{hmdC}, 5 \mathrm{fdC}$ and $5 \mathrm{cadC}$ in gDNA from 3D7-wild type (WT) parasites versus gDNA from the two Pf-DNMT2KO clones cl1 and cl2. Data is shown as percentage of modified deoxycytidines relative to the total number of deoxycytidines in each sample and represents the mean $( \pm S D)$ of two independent replicates of each $\operatorname{strain}(n=2)$.

C. DNMT2-SNAP expression in S. Cerevisiae cells. Scale bar $=10 \mu \mathrm{m}$. DIC: differential interference contrast; Hoechst: nuclear staining.

D. Immuno dot-blot analysis of $5 \mathrm{mC}$ in gDNA from yeast cells non-transformed (NT) or transformed (T) with Pf-DNMT2-SNAP plasmid.

E. LC-MS/MS-based quantification of $5 \mathrm{mdC}$ in gDNA from yeast cells nontransformed (NT) or transformed (T) with Pf-DNMT2-SNAP plasmid.

Statistical analysis was carried out using 2way ANOVA. P Value $<0.0001={ }^{* * * *}$; $\mathrm{P}$ Value $<0.005{ }^{* *}$

\section{Supplementary figures legends}

Figure S1: LC-MS/MS chromatogram of deoxycytidine (dC), 5-methyl-2'deoxycytidine (5mdC), 5-hydroxymethyl-2'-deoxycytidine (5hmdC), 5-carboxy-2'deoxycytidine (5cadC), and 5'-formyl-2'-deoxycytidine (5fdC) in A) mESC gDNA and B) P. falciparum gDNA according to the digestion protocol. Blue arrows show the expected 5hmdC peak while the red arrows indicate the shifted 5hmdC peak observed earlier ${ }^{23}$. 
Figure S2: In vitro cultured parasite growth under normal $\left(5 \% \mathrm{O}_{2}\right)$ and high oxygen concentrations $\left(20 \% \mathrm{O}_{2}\right)$.

Giemsa staining (A) and DNA quantification (B) during the 48h blood stage cycle do not reveal any developmental changes between parasites cultured at $5 \%$ vs $20 \% \mathrm{O}_{2}$.

Figure S3: LC-MS/MS quantification of $5 \mathrm{mdC}, 5 \mathrm{hmdC}, 5 \mathrm{fdC}$ and $5 \mathrm{cadC}$ in gDNA of DNMT2KO parasite from ring (A) and schizont stage (B).

Nucleoside quantification of $5 \mathrm{mdC}, 5 \mathrm{hmdC}, 5 \mathrm{fdC}$ and $5 \mathrm{cadC}$ in gDNA from 3D7-wild type (WT) parasites versus gDNA from the two Pf-DNMT2KO clones cl1 and cl2. Data is shown as percentage of modified deoxycytidines relative to the total number of deoxycytidine in each sample and represents the mean $( \pm S D)$ of two independent replicates of each strain. 